\title{
Research on the attenuation characteristics of some inorganic salts in seawater
}

X. Han

Y. Peng

yufengp@htu.cn

Y. Zhang

Z. Ma

J. Wang
College of Physics and Electronic Engineering, Henan Normal University, Xinxiang 453007, China

College of Physics and Electronic Engineering, Henan Normal University, Xinxiang 453007, China

College of Physics and Electronic Engineering, Henan Normal University, Xinxiang 453007, China

The 404 Company Limited, China National Nuclear Corporation, Lanzhou, 735112, China

Seawater is a complex multicomponent system, which involves varieties of organic, inorganic, dissolved and suspended substances. However, the main components dissolved in seawater are the inorganic salts such as $\mathrm{NaCl}, \mathrm{MgCl}, \mathrm{KCl}_{1} \mathrm{NaHCO}_{3}$, and $\mathrm{MgSO}_{4}$. These elements make different contributions to the spectra of absorption and scattering in water. In this paper, the spectra of different aqueous solutions were measured in the region from 200 to $1200 \mathrm{~nm}$; the attenuation characteristics of aqueous solutions were studied at wavelengths of 450, 532, and $633 \mathrm{~nm}$, respectively; the relationships between attenuation coefficient and the conductivity in different concentrations were also studied.

[DOI: http://dx.doi.org/10.2971/jeos.2015.15045]

Keywords: Seawater, inorganic salts, spectrum, attenuation coefficient, conductivity

\section{INTRODUCTION}

The development of research on high-energy laser technology, underwater optical communication, seabed imaging, etc, has generated interest for many scholars [1]-[4]. Due to the scattering and absorption of light in seawater, the underwater optical signal attenuation is relatively large. In 1963, Sullivan and Dimtle discovered the low-loss "optical window" of undersea communication for blue-green lasers between $420-532 \mathrm{~nm}$. Since then, research on underwater laser communication technology has not stopped [5]-[7].

In seawater, the main dissolved substances are inorganic salts, such as $\mathrm{NaCl}, \mathrm{MgCl}_{2}, \mathrm{KCl}, \mathrm{NaHCO}_{3}, \mathrm{MgSO}_{4}$, and so on. Several authors have reported the optical properties of seawater. Morel $[8,9]$ has measured the scattering of $\mathrm{NaCl}$ solutions whose concentration is up to $100 \mathrm{~g} / \mathrm{L}$ at $366 \mathrm{~nm}$ and $546 \mathrm{~nm}$. Ravisankar and Reghunath [10] have measured the effect of dissolved $\mathrm{NaCl}, \mathrm{MgCl}_{2}$, and $\mathrm{Na}_{2} \mathrm{SO}_{4}$ in seawater on the optical attenuation in the region from $430 \mathrm{~nm}$ to $630 \mathrm{~nm}$ by using the split-pulse method. Compared to the attenuation values of natural and artificial seawater, they found that the changes of attenuation value in the wavelength range from 430 to $630 \mathrm{~nm}$ have no clear relationship with the concentration of dissolved substances, but with the suspended particles in the solution. Pegau [11] studied the attenuation of solutions in different salinity in the visible and near-infrared regions. Based on the study of the effect of salinity on the dielectric properties of water by Gadani [12], the permittivity was found to decrease with increasing salinity and the relationship between emissivity of water and the salinity had the same rule, but the dielectric loss was found to increase with the salinity. Recently, Zhang et al. [13] deduced the effect of sea salts on the thermodynamics of scattering by seawater and found that scattering increased non-linearly with salinity.

Although there are some differences in the estimates of optical properties, the connection between theory and experiment is well developed. In contrast, our theoretical and experimental understanding of absorption and attenuation coefficients by water and solutions is quite limited [11].

In this paper, the effect of the concentration of these inorganic salts on attenuation is investigated by UV-spectrophotometry. Simultaneously, the conductivity of each solution was measured by a conductivity meter. During the measurement of conductivity, temperature compensation was used to reduce the error rate caused by the temperature of the experiment. The main purpose of this study is to identify the relationship between conductivity and the optical attenuation, and thereby to analyze the optical properties of seawater. The work in this paper is helpful for related research. 


\section{EXTINCTION MECHANISM}

The optical characters of seawater are easy to change, mainly because of the variability of dissolved substances and suspended particles $[14,15]$. There are two distinct causes for the energy loss of a light signal in seawater: one is absorption and the other is scattering.

dris the incremental path length for monochromatic collimating light transmission in seawater. The loss of luminous flux caused by absorption is $d \Phi=-a \Phi d r$ and by scattering is $d \Phi=-b \Phi d r$. The proportional coefficient $a$ is the whole absorption coefficient, and $b$ is the whole scattering coefficient [16].

The absorption coefficient of seawater can be expressed as follows:

$$
a(\lambda)=a_{w}(\lambda)+a_{p} h(\lambda)+a_{C D O M}(\lambda)+a_{d}(\lambda)
$$

The scattering coefficient of seawater can be expressed by:

$$
b(\lambda)=b_{w}(\lambda)+b_{p h}(\lambda)+b_{d}(\lambda)
$$

where the subscripts $w, p h, C D O M$, and $d$ represent the absorption of water molecules, phytoplankton (chlorophyll), color dissolved organic matter and other dissolved material respectively.

The total attenuation coefficient can be expressed as

$$
c(\lambda)=a(\lambda)+b(\lambda)
$$

\section{EXPERIMENTAL}

\subsection{Solution preparation}

The water used in this study was high-level purified water, which was produced by a Milli-Q Millipore System (Milli-Q Advantage A10) and had a resistivity of about $18.2 \mathrm{M} \Omega \cdot \mathrm{cm}$ at $298 \mathrm{~K}$. The total organic carbon and dissolved oxygen contents of the water were below $0.05 \mathrm{mg} / \mathrm{L}$ and $5.0 \mathrm{mg} / \mathrm{L}$, respectively.

All the inorganic salts $\mathrm{NaCl}, \mathrm{MgCl}_{2}, \mathrm{KCI}, \mathrm{NaHCO}_{3}$, and $\mathrm{MgSO}_{4}$ were of p.a. grade from Tianjin Kermel chemical reagent Co., Ltd, China and were used as received. They were weighed by an electronic balance (ALC-110.4). All the solutions were prepared using high-level purified water, corresponding to concentrations in the range from $0.8 \%$ to $50 \%$. In order to reduce the experimental error, solutions were gradually doubly diluted from high concentration to low concentration. All solutions were stored in polyethylene flasks to avoid any contamination coming from glass surfaces.

\subsection{Measurements}

An UV-visible spectrophotometer (UV-3600230VCE) was used for the absorption spectroscopy determination of solutions, over a wavelength scan range from $200 \mathrm{~nm}$ to $1200 \mathrm{~nm}$. The solution was placed in a cylindrical cuvette with a length of $10 \mathrm{~cm}$ and bottom diameter of $1.8 \mathrm{~cm}$. After measuring each solution, in order to prevent the contamination by other solutes, the cuvette must firstly be cleaned three times using distilled water, and then be subjected to a low pressure plasma treatment system for $5 \mathrm{~min}$. Because the whole process of cleaning and drying is automatically completed in the plasma cleaner, the cuvette can be used directly after being cleaned.

The conductivity was measured using a conductivity meter whose measurement range was $0-2 \times 10^{5} \mu \mathrm{s} \cdot \mathrm{cm}^{-1}$, accuracy was $\pm 1 \%$ F.S, instrument stability was $0.5 \%$, and temperature compensation range was $15-35^{\circ}$. There were no significant vibrations or strong magnetic field during measurement, in strict compliance with the prescribed working conditions of the instrument. The conductivity of each solution with a different concentration was measured for $5 \mathrm{~min}$ and repeated five times to eliminate error.

\section{RESULTS AND DISCUSSION}

\subsection{Relationship between attenuation and wavelength}

The attenuation of different solutions $\left(\mathrm{NaCl}, \mathrm{MgCl}_{2}, \mathrm{KCI}\right.$, $\mathrm{NaHCO}_{3}$, and $\mathrm{MgSO}_{4}$ ) with different concentrations measured by UV-spectrophotometer are shown in Figure 1.

From Figure 1, it can be seen that different solutions with different concentrations correspond to different values of the absorption, but the shapes of the attenuation curves are approximately the same and the wavelength range of minimum attenuation does not change. It is easy to observe that the minimum attenuation values are mainly concentrated in the range from 450-633 $\mathrm{nm}$ and that in this area, the attenuation values increase with the concentration of the aqueous solutions. According to the attenuation theory $[11,17]$, in the visible spectrum, the shorter the wavelength is, the stronger the scattering by water molecules is and the absorption can be ignored. So, in the visible region, the total attenuation is more obvious at shorter wavelengths than at longer wavelength and the conclusion is validated by this experiment.

\subsection{Relationship between attenuation and conductivity}

The conductivity of the solutions obtained using first-order index curve fitting. The relationships between the concentration and the conductivities of solutions are shown below.

From Figure 2, it is evident that the relationship between conductivity and concentration is nonlinear. The curves show that the conductivity decreases with decreasing concentration; the conductivities of the solutions with different concentrations are different; the $\mathrm{NaCl}$ solution has maximum conductivity and the $\mathrm{NaHCO}_{3}$ solution has the minimum. This may be related to the varying effects of different ions on the structure of water [18].

In order to analyze the relationship between the attenuation coefficient and the conductivity, we measured the conductiv- 


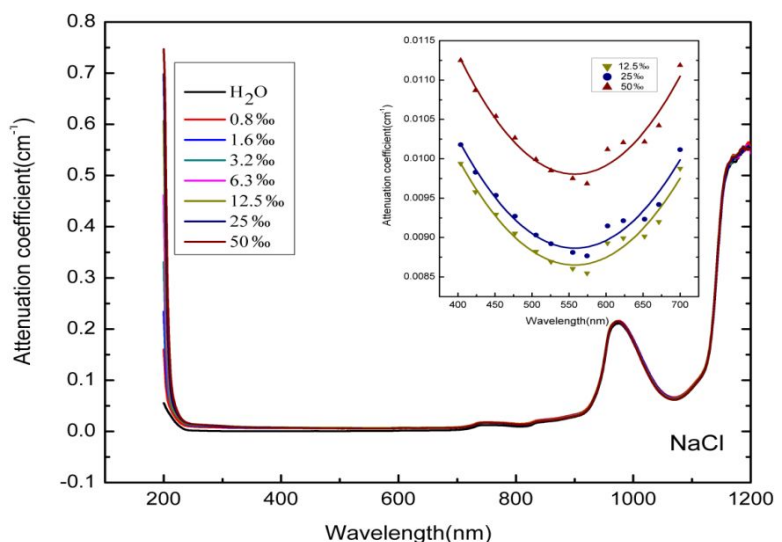

(a)

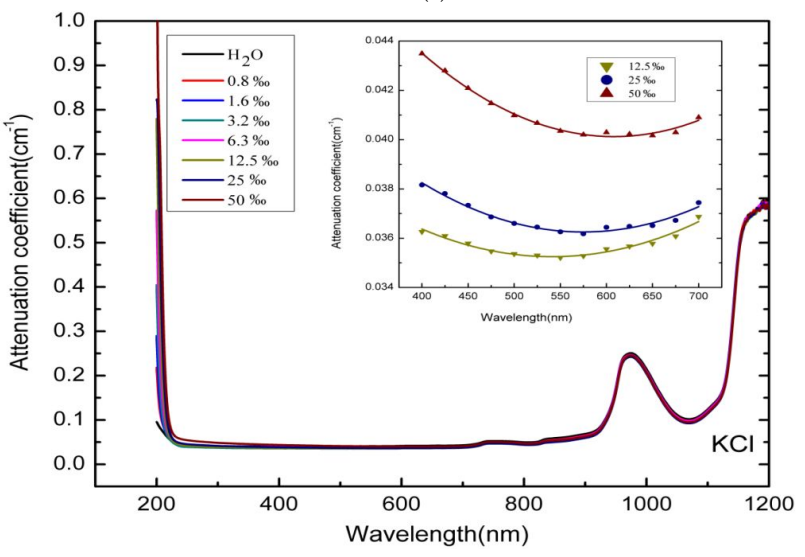

(c)

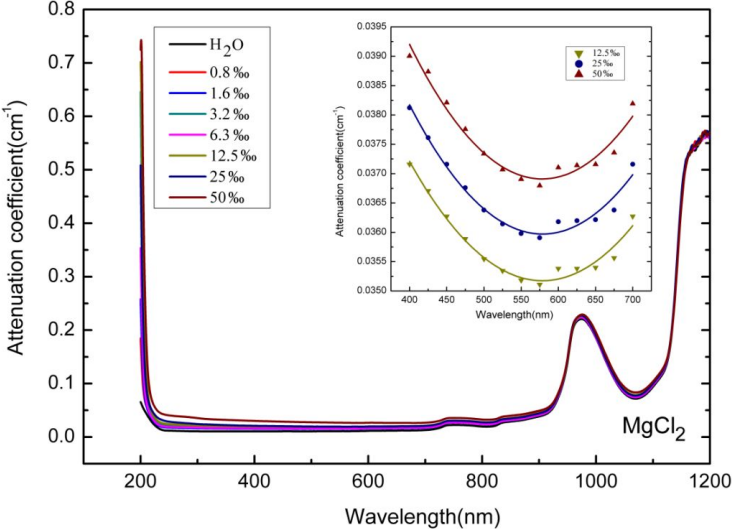

(b)

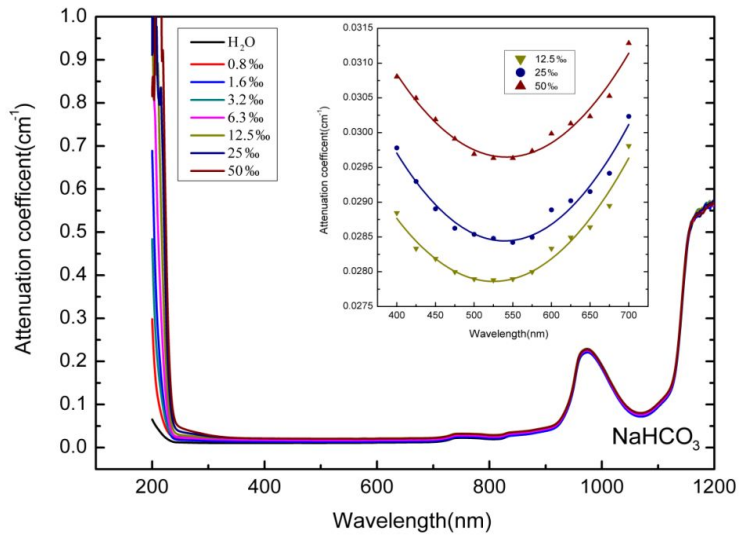

(d)

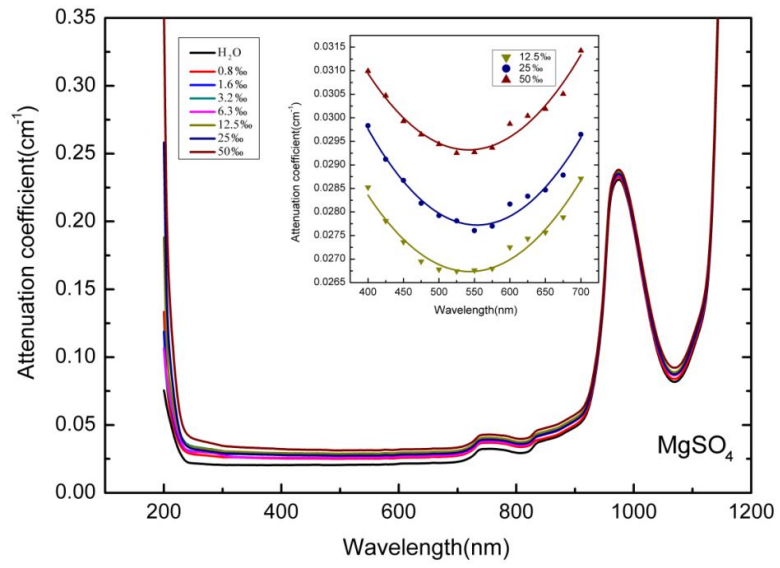

(e)

FIC. 1 The relationships between attenuation and wavelength for inorganic salt solutions with different concentrations, (a) $\mathrm{NaCl}$ (b) $\mathrm{MgCl}_{2}$; (c) $\mathrm{KCl}$ (d) $\mathrm{NaHCO}_{3}$; (e) $\mathrm{MgSO}_{4}$ The enlarged curves in the insets show the spectra in the visible wavelength region.

ity of the solutions with the same solute at particular wavelengths (450 nm, $532 \mathrm{~nm}$ and $633 \mathrm{~nm}$ ). The same method was used for all the solutions in this experiment. The simulation curves for the relationship between attenuation and conductivity are shown in Figure 3 and the fitting function can be expressed as

$$
A=A_{0}+B e^{-x / t}
$$

The following are the parameters of the first-order index fitting of Eq. (4) corresponding to the three different wavelengths. These results provide an available reference for later research on attenuation of seawater.

As shown in Figure 3, the attenuation values of different solutions are related to the conductivity. The regularity of the

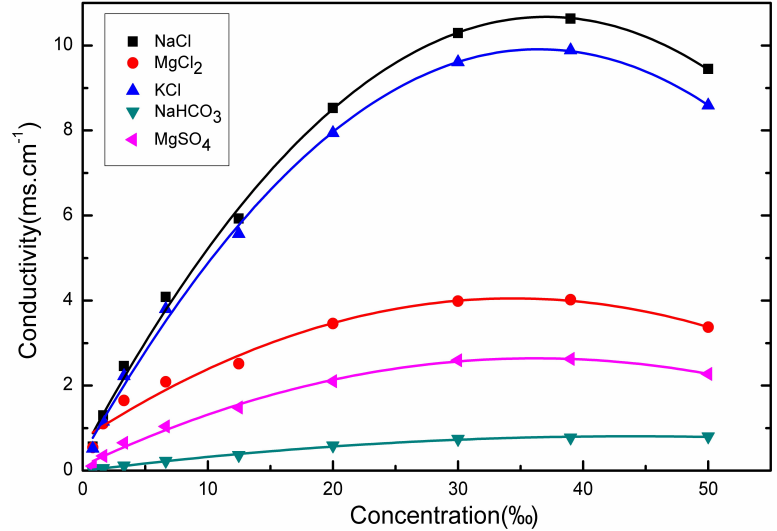

FIG. 2 Relationships between concentration and conductivity. 


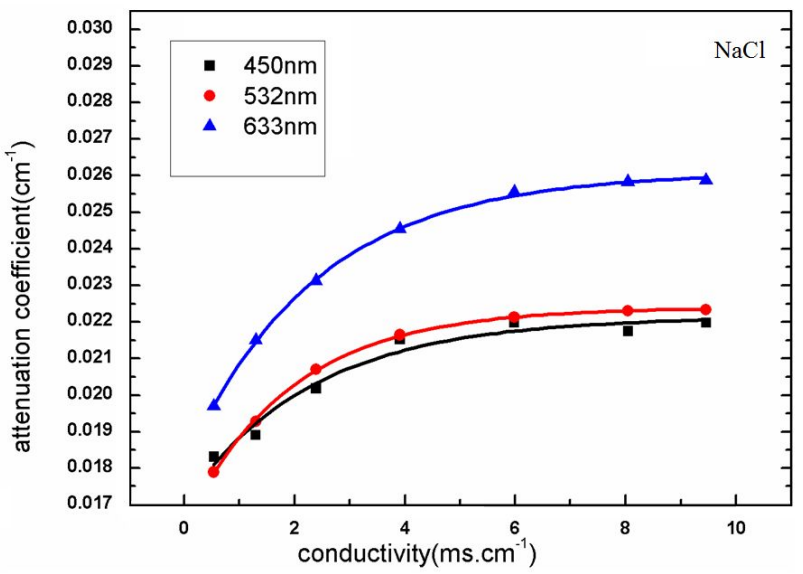

(a)

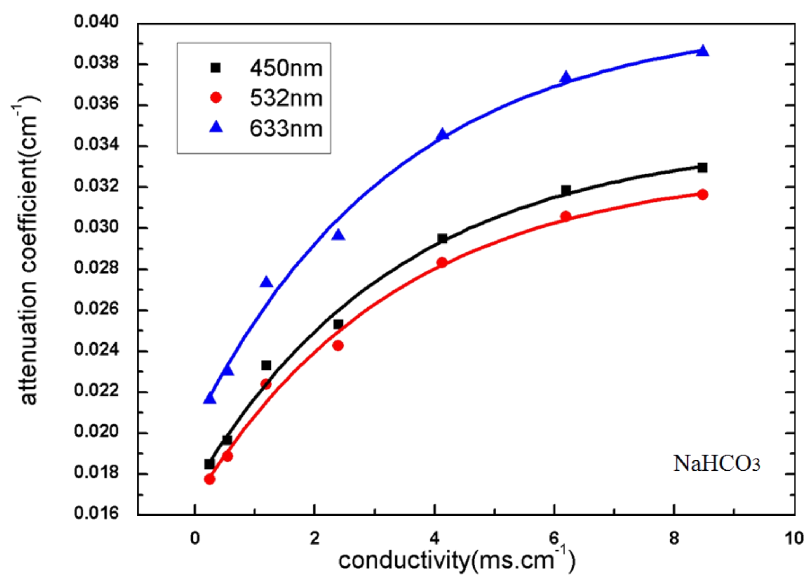

(c)

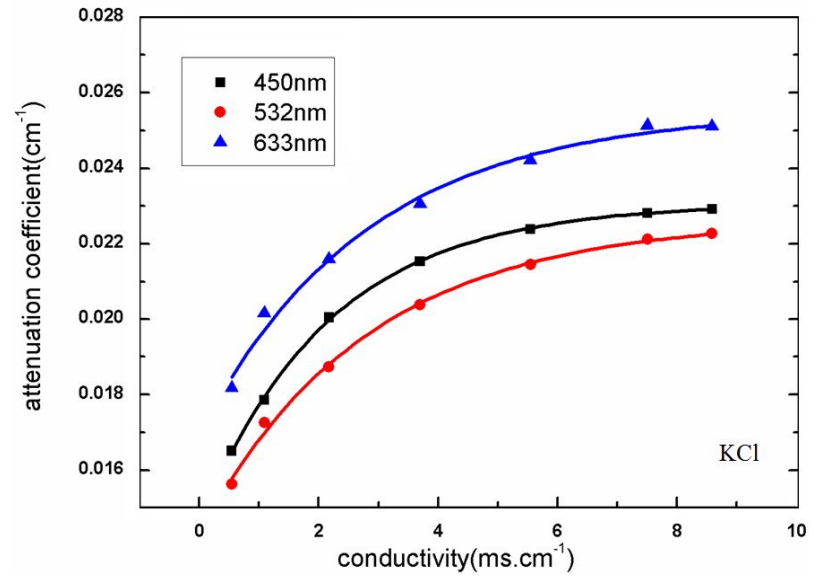

(b)

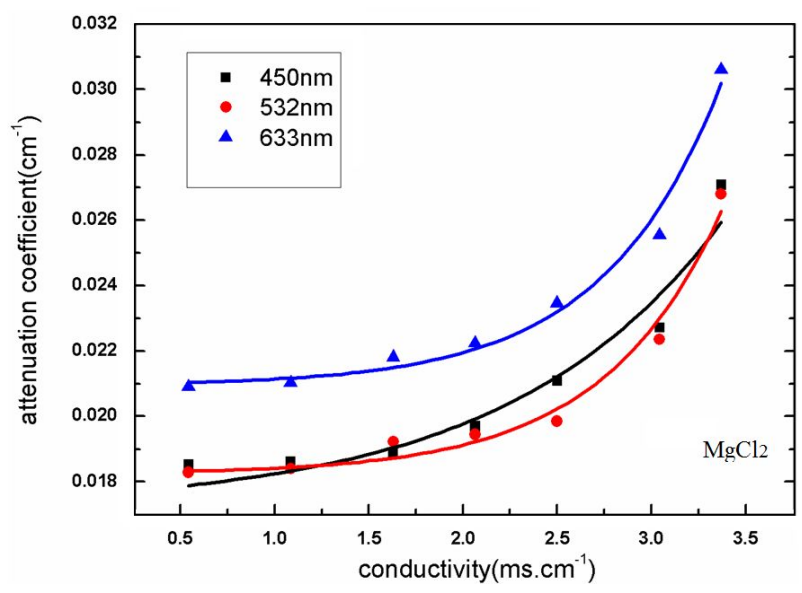

(d)

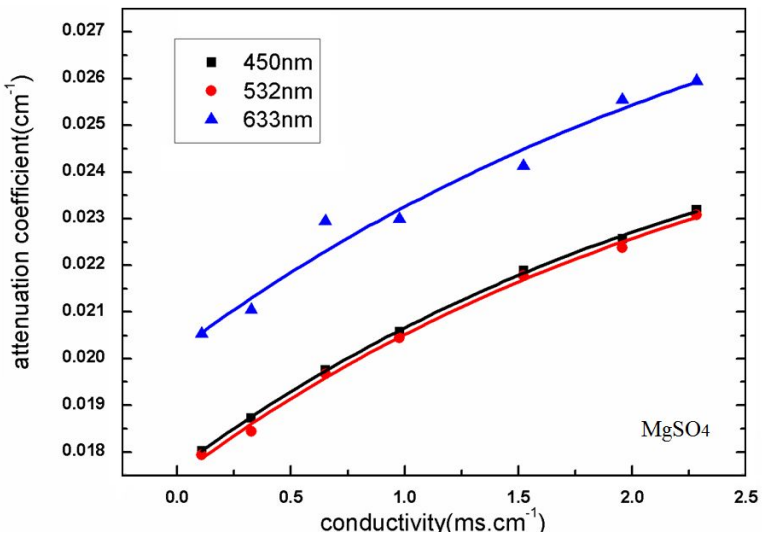

(e)

FIG. 3 The relationships between attenuation coefficient and conductivity. (a) $\mathrm{NaCl}$; (b) $\mathrm{KCl}$; (c) $\mathrm{NaHCO}_{3}$; (d) $\mathrm{MgCl}_{2}$; (e) $\mathrm{MgSO}_{4}$.

\begin{tabular}{|c|c|c|c|c|c|c|}
\hline & & $\mathrm{NaCl}$ & $\mathrm{MgCl}_{2}$ & $\mathrm{KCl}$ & $\mathrm{NaHCO}_{3}$ & $\mathrm{MgSO}_{4}$ \\
\hline \multirow{3}{*}{$450 \mathrm{~nm}$} & $A_{0}$ & 0.02214 & 0.0172 & 0.02307 & 0.03444 & 0.02698 \\
\cline { 2 - 7 } & $B$ & -0.00509 & 0.00044 & -0.00849 & -0.01705 & -0.00935 \\
\cline { 2 - 7 } & $t$ & 2.31843 & -1.12568 & 2.15311 & 3.401 & 2.54746 \\
\hline \multirow{3}{*}{$532 \mathrm{~nm}$} & $A_{0}$ & 0.02239 & 0.01826 & 0.02264 & 0.03308 & 0.02682 \\
\cline { 2 - 7 } & $B$ & -0.00601 & 0.00004 & -0.00834 & -0.01638 & -0.00935 \\
\cline { 2 - 7 } & $t$ & 1.90827 & -0.62345 & 2.79656 & 3.40116 & 2.52978 \\
\hline \multirow{3}{*}{$633 \mathrm{~nm}$} & $A_{0}$ & 0.02609 & 0.02097 & 0.02553 & 0.04036 & 0.03065 \\
\cline { 2 - 7 } & $B$ & -0.00802 & 0.00004 & -0.00857 & -0.01998 & -0.01048 \\
\cline { 2 - 7 } & $t$ & 2.36146 & -0.61777 & 2.79771 & 3.40116 & 2.86315 \\
\hline
\end{tabular}

TABLE 1 The fitting parameters for the attenuation coefficients of different solutions. 
curves shows that the attenuation decreases with decreasing conductivity.

The above phenomenona may be influenced by the following factors: the solutions used in this experiment were mostly ionic. When the concentration of the solution was diluted slowly from the high concentration, the charged body of the solution per unit volume decreased, and the effect of the laser energy on the by charged body decreased and so the attenuation decreased with decreasing conductivity. The results are in agreement with Beer-Lambert law, which indicates that the experimental results are reliable.

\section{CONCLUSIONS}

Optical attenuation coefficients have been determined for different concentrations of $\mathrm{NaCl}, \mathrm{MgCl}_{2}, \mathrm{KCl}, \mathrm{NaHCO}_{3}$, and $\mathrm{MgSO}_{4}$ in water. The attenuation spectra of different solutions with different concentrations have been studied and it has been shown that the attenuation values decrease with decreasing concentration in the visible wavelength region. The relationships between the attenuation and the conductivity have been analyzed and indicate that the conductivity of the solution is related to the concentration and that the conductivity decreases with decreasing concentration. The attenuation also decreases with decreasing conductivity, which may be related to the electronic structure of the ions in solution, especially for the magnesium salts. It should be worthwhile to conduct further research on the characteristic features of optical propagation in sea-water and their origins.

\section{ACKN OWLEDGEMENTS}

We wish to thank the National Natural Science Foundation of China (Grant No. 5201029120032) for the opportunity to do the resulting research.We are also grateful for the comments made by the anonymous reviewer.

\section{References}

[1] V. I. Man'kovsky, "Spectral contributions of the components of seawater to the beam attenuation coefficient in surface waters of the Mediterranean Sea," Physical Oceanography 21, 305-319 (2012).

[2] F. Hanson, and M. Lasher, "Effects of underwater turbulence on laser beam propagation and coupling into single-mode optical fiber," Appl. Optics 49, 3224-3230 (2010).

[3] H. T. Yura, "Propagation of finite cross-section laser beams in sea water," Appl. Optics 12, 108-115 (1973).
[4] E. Y. S. Young, and A. M. Bullock, "Underwater-airborne laser communication system: characterization of the channel," Proc. SPIE 4975, 146-157 (2003).

[5] A. N. Z. Rashed, and H. A. Sharshar, "Performance evaluation of short range underwater optical wireless communications for different ocean water types," Wireless Pers. Commun. 72, 693-708 (2013).

[6] E. Kazemian, and F. D. Kashani, "Reliability analysis of underwater optical communication links in different locations through Caspian Sea," Optik 124, 5184-5188(2013).

[7] A. T. Reghunath, V. Venkataramanan, D. V. Suviseshamuthu, R. Krishnamohan, and B. R. Prasad, "The origin of blue-green window and the propagation of radiation in ocean waters," Defence Sci. J. 41, 1-20 (2013).

[8] A. Morel, "Etude expérimentale de la diffusion de la lumiére par l'eau, les solutions de chlorure de sodium, et l'eau de mer optiquement pures," J. Chim. Phys. 10, 1359-1366 (1966).

[9] A. Morel, D. Antoine, and B. Gentili, "Bidirectional reflectance of oceanic waters: accounting for Raman emission and varying particle scattering phase function," Appl. Optics 41, 6289-6306 (2002).

[10] M. Ravisankar, and A. T. Reghunath, "Effect of dissolved $\mathrm{NaCl}$, $\mathrm{MgCl}_{2}$, and $\mathrm{Na}_{2} \mathrm{SO}_{4}$ in sea water on the optical attenuation in the region from 430 to $630 \mathrm{~nm}$," Appl. Optics 27, 3887-3894 (1988).

[11] W. S. Pegau, D. Gray, and J. R. V. Zaneveld, "Absorption and attenuation of visible and near-infrared light in water: dependence on temperature and salinity," Appl. Optics 36, 6035-6046 (1997).

[12] D. H. Gadani, V. A. Rana, S. P. Bhatnagar, A. N. Prajapati, and A. D. Vyas, "Effect of salinity on the dielectric properties of water," Indian J. Pure Ap. Phys. 50, 405-410 (2012).

[13] X. Zhang, and L. Hu, "Scattering by pure seawater at high salinity," Opt. Express 17, 12685-12691 (2009).

[14] D. Stramski, E. Boss, D. Bogucki, and K. J. Voss, "The role of seawater constituents in light back scattering in the ocean," Prog. Oceanogr. 61, 27-56 (2004).

[15] A. H. Hakim, and N. J. McCormick, "Ocean optics estimation for absorption, backscattering, and phase function parameters," Appl. Optics 42, 931-938 (2003).

[16] M. Stramska, and M. Świrgoń, "Influence of atmospheric forcing and freshwater discharge on interannual variability of the vertical diffuse attenuation coefficient at $490 \mathrm{~nm}$ in the Baltic Sea," Remote Sens. Environ. 140, 155-164 (2014).

[17] J. M. Sullivan, M. S. Twardowski, J. R. V. Zaneveld, C. M. Moore, A. H. Barnard, P. L. Donaghay, and B. Rhoades, "Hyperspectral temperature and salt dependencies of absorption by water and heavy water in the $400-750 \mathrm{~nm}$ spectral range," Appl. Optics 45 , 5294-5309 (2006).

[18] Y. Marcus, "A simple empirical model describing the thermodynamics of hydration of ions of widely varying charges, sizes, and shapes," Biophys. Chem. 51, 111-127 (1994). 